\title{
Comparative effect of natural commodities and commercial medicines against oral thrush causing fungal organism of Candida albicans
}

\author{
Reena, $T^{1}$, Rohitha Prem ${ }^{1}$, Deepthi, M. S ${ }^{1}$, R. Beni Ramachanran ${ }^{1}$, S. Sujatha ${ }^{2, *}$ \\ ${ }^{1}$ Department of Microbiology, Malanakara Catholic College, Mariagiri, Kaliakkavilai \\ ${ }^{2}$ International Centre for Bioresources Management, Malanakara Catholic College, Mariagiri, Kaliakkavilai
}

\section{Email address:}

sujatharbs@rediffmail.com(S. Sujatha)

\section{To cite this article:}

Reena, T, Rohitha Prem, Deepthi, M. S, R. Beni Ramachanran, S. Sujatha. Comparative Effect of Natural Commodities and Commercial Medicines Against Oral Thrush Causing Fungal Organism of Candida Albicans. Science Journal of Clinical Medicine. Vol. 2, No. 3, 2013, pp. 75-80. doi: 10.11648/j.sjcm.20130203.13

\begin{abstract}
The aim of the study was to explore the comparative analysis antifungal efficiency of six natural commodities and four commercial medicines against the oral thrush causing organism of Candida albicans. From the present result along with the six natural commodities, Mayaca showed maximum inhibitory activity against C. albicans followed by garlic, gooseberry, wine, coconut oil and pomegranate. While, the significant antifungal activity noted in Mayaca ehtanolic extract against $\mathrm{C}$. albicans at 50 and $100 \mu$ concentration $(\mathrm{P}<0.05)$, and other natural substances such as garlic and gooseberry antifungal activity also expressed significantly. In the GC-MS analysis ten bioactive compounds were identified in the ehtanolic extract. Besides the identified bioactive peak phytochemical compounds named as 3,4-Dimethyl-2-3-methyl with its Ret. time 19.050 followed by second and third peak compounds are Diethylpthalate and Bis-3,4 methylene Dioxy accompanied with them responsible RT was 21.004 and 28.666 respectively. The overall results clearly denoted ethanol extract of Mayaca act as significant antifungal C. albicans agent mainly it was possessed specific antimicrobial secondary metabolic compounds present than other five natural commodities and four commercial products. Hence, the present study focused that the Mayaca extract act as a potential antifungal agent for oral thrush causing fungi of C. albicans.
\end{abstract}

Keywords: Candida Albicans, Natural Commodities, Mayaca, GC-MS

\section{Introduction}

Oral candidiasis is caused by an overgrowth or infection of the oral cavity by a yeast-like fungus, Candida. The most common are $C$. albicans followed by $C$. tropicalis, $C$. glabrata, C. pseudotropicalis, C. guillierimondii, C. krusei, C. lusitaniae, C. parapsilosis, and C. stellatoidea. ${ }^{1}$ The disease is typically limited to infants and neonates, patients on antibiotics or steroids, and patients with polyendocrine disorders or underlying immune dysfunction to Children of oral candidiasis. ${ }^{2}$ The increasing interest on traditional medicine may lead to discovery of novel therapeutic agents. Natural products of higher plants may offer a new source of antimicrobial agents for external use. ${ }^{3}$

In the plant kingdom, there are thousands of plants known and unknown that yield medicine or drugs use to man these plants and its seeds were known as medicinal or drug plants, Medicinal phytochemical compounds (quantitative method) using plants are at great interest to the researcher in the field of GC-MS analysis. Biotechnology, as most of the drug industries depend in part on plants for the production of pharmaceutical ${ }^{4}$ Medicinal phytochemical compounds (quantitative method) using plants are at great interest to the researcher in the field of GC-MS analysis ${ }^{5}$. The antifungal activity of propolis has been specifically evaluated against different fungi. The genera Aspergillus ${ }^{6}$, Candida $^{7}$ yeasts. $^{8,} 9$ and others have been analyzed regarding their susceptibility to propolis or to some of its components. These and other analyses have determined that pinocembrin, galangin and pinobanksin are the predominant compounds in the studied propolis. ${ }^{10}$. Candida albicans generally grows as yeast at $\leq$ $30^{\circ} \mathrm{C}$ and as filaments at $37^{\circ} \mathrm{C}$ with regards to quorum sensing, and it can be produced also secrete an extracellular molecule, farnesol, which regulates the morphological transition. ${ }^{11}$ 
There are several reports that show antifungal activity by natural products against oral, intestinal and food-borne bacteria, antitoxicity against various bacterial haemolysins and antiviral activity ${ }^{12}$. Still today no other works has been done this kind of similar works hence the present study designed the following objectives to evaluate the anticandidal (antifungal) effect of natural commodities and commercial drugs and to study the proportional of inhibitory effect natural and commercial drugs against the fungal organisms (Candida albicans). Subsequently to determine the photochemical compounds (quantitative method) using GC-MS analysis.

\section{Materials and Methods}

\subsection{Sample Collection}

This study was conducted in Department of Microbiology at Malankara Catholic College, Mariagiri from December-2010- May 2011.

Oral thrush samples were collected from babies (below one year) of Trivandrum district, Kerala. Thirty samples were collected aseptically using sterile swabs in a screw cap tubes and brought to the laboratory in ice box and stored at $4{ }^{\circ} \mathrm{C}$ in refrigerator for further studies.

\subsection{Collection of Natural Commodities}

Six different Indian natural commodities such as mayacca, garlic, wine, coconut oil, pomegranate and gooseberry were collected from local shops.

\subsection{Isolation of Candida Species}

The oral thrush samples in swabs were streaked on Sabouraud Dextrose agar plates aseptically. The plates were then incubated at $37^{\circ} \mathrm{C}$ in inverted position for 24 hours. After the incubation, plates were observed and the isolated colonies were separated for the purification and identification and the results were recorded. The isolated colonies were continuously streaked on Sabouraud Dextrose agar and incubated at $37^{\circ} \mathrm{C}$ for 24 hours for purification.

\subsection{Preparation of Extracts}

Six different natural extracts were prepared by crushing and dissolving in ethanol solvent. These extracts were kept for 20 to 30 days. Then these extracts were taken for the further anticandidal activity against oral thrush pathogens.

\subsection{Testing of Antifungal Activity (Kirby-Bauer Method) Well Diffusion Method}

Muller-Hinton agar were sterilized and poured into sterile petriplates. The Candida species were swabed on the agar plates. By using well cutter, each well the extracts were poured and tested for the antifungal activity. Formation of zone indicated the positive result ${ }^{13}$.

\subsection{Disc Diffusion Method}

The Candida species were swabed on Muller-Hinton agar plates and the antibiotic discs were placed on the agar. Each disc on the agar surface was gently pressed down and the plates were incubated in an inverted position for 24 hours at $37^{\circ} \mathrm{C}$. After incubation the results were recorded in $\mathrm{mm}^{14}$.

\subsection{Determination of MIC}

Minimum inhibitory method was applied on extracts that proved their high efficacy against microorganism. Selected plant extracts were subjected to serial dilution using sterile Muller-Hinton broth medium. Added one $\mathrm{ml}$ of culture to each tube and incubated for 18 hours at $37^{\circ} \mathrm{C}$. The highest dilution of the plant extract that retained its inhibitory effect resulting in no growth (absence of turbidity) of a microorganism was as the MIC value of the extract

\subsection{Gas Chromatography Mass-Spectrometry Analysis (GC-MS)}

The identification of constituents was performed by a Hewlett Packard gas chromatograph 6890 series II Plus linked to Hewlett Packard mass spectrometer system equipped with a capillary column HP5-MS (30 m/0.25 mm, $0.25 \mu \mathrm{m}$ film thickness). The temperature was programmed from $23^{0^{\circ}} \mathrm{C}$ to $30^{0^{\circ}} \mathrm{C}$ at a rate of $4^{\circ} \mathrm{C}$ minD1 with $10 \mathrm{~min}$ hold. Injector was at $28^{0^{\circ}} \mathrm{C}$. Helium was used as a carrier gas with a constant flow at $0.8 \mathrm{ml}$ minD1. The ionization voltage was $70 \mathrm{eV}$. Fraction was analyzed also after silylation at the conditions given for the silylated polar compounds mentioned below. Quantitative analysis was performed on a Hewlett Packard gas chromatograph 5890 equipped with FID and capillary column HP5-MS (30 $\mathrm{m} / 0.25 \mathrm{~mm}, 0.25 \mu \mathrm{m}$ film thickness), at $230^{\circ} \mathrm{C}$ and programmed to $300^{\circ} \mathrm{C}$ at $4^{\circ} \mathrm{C} \operatorname{minD} 1$ and 10 min hold. Injector and detector were at $280^{\circ} \mathrm{C}$. One $\mu$ l of each sample were injected triplicate split/spiltless and quantities represented as relative area $\%$ as derived from the integrator.

\subsection{Statistical Analysis}

The results were compared for the effectiveness of natural and commercial products. ANOVA test (Analysis of Variance): Analysis of variance is a statistical method used to test whether the effects of several factors are equal or not. The ANOVA is designed to test whether a significant difference exists among the analyzed data

\section{Results}

\subsection{Antifungal Effect of Plant Extract}

The antifungal activity of Mayaca, Garlic, Pomegranate, Gooseberry, Wine, and Coconut oil in ethanolic extract against Candida albicans were tabulated (Table-1). Among 
the six natural commodities, Mayaca showed maximum inhibitory activity against $C$. albicans followed by garlic, gooseberry, wine, coconut oil and pomegranate. Among the commercial drugs, nystatin showed high effectiveness followed by amphotericin-B, fluconazole and miconazole.

Table 1. Natural Commodities and its Ethanol Extract against the Zone of Inhibition (in Diameter) of Candida albicans.

\begin{tabular}{llccc}
\hline SI No: & $\begin{array}{l}\text { Natural commodities } \\
\text { ethanol extract }\end{array}$ & \multicolumn{3}{c}{ Zone of Inhibition $(\boldsymbol{\mu l})$} \\
\hline 1. & Mayaca & $\mathbf{5 0} \mathbf{~ m m}$ & $\mathbf{1 0 0 \mathbf { m m }}$ & $\mathbf{1 5 0} \mathbf{~ \mathbf { m }}$ \\
2. & Garlic & 5 & 15 & 25 \\
3. & Gooseberry & 10 & 16 & 18 \\
4. & Wine & 6 & 9 & 12 \\
5. & Coconut oil & - & - & 2 \\
6. & Pomegranate & 4 & 5 & 9 \\
\hline
\end{tabular}

Similarly three different concentrations also noted Mayaca shows peak activity against the $C$. albicans compaered with other five natural commodities.

From the table-2 showed the result of zone of inhibition performance of six natural commodities and its ehtanolic extract on C. albicans. From this table indicated that the maximum zone of inhibition $25 \mathrm{~mm}$ identified in Mayaca extract at the notable concentration was $150 \mu \mathrm{l}$. While the other higher ZI observed on Garlic 19 and Gooseberry $18 \mathrm{~mm}$ at similar concentration. In addition very least activity had been noticed in coconut oil.

Table 2. MIC value of natural extract against the oral thrush causing $C$. albicans.

\begin{tabular}{lcccccc}
\hline Name of the Samples & \multicolumn{5}{c}{ Concentration $(\boldsymbol{\mu l})$} \\
& $\mathbf{5 0}$ & $\mathbf{1 0 0}$ & $\mathbf{1 5 0}$ & $\mathbf{2 0 0}$ & $\mathbf{2 5 0}$ \\
\hline Mayaca extract & $0.95 * *$ & 0.50 & 0.44 & 0.34 & 0.14 is \\
Gooseberry fruit & 0.64 & $0.63 * *$ & 0.59 & $0.26 \mathrm{is}$ & 0.14 \\
Garlic extract & $0.90 * *$ & $0.89 *$ & $0.64 *$ & 0.29 is & 0.22 is \\
Wine & $0.74 *$ & 0.51 & $0.56 *$ & 0.53 & 0.60 \\
Coconut oil & 0.53 & 0.51 & 0.27 is & 0.40 & 0.36 \\
Pomegranate fruit juice & 0.79 is & $0.75^{*}$ & 0.73 & $0.54 *$ & 0.49 \\
\hline
\end{tabular}

**-Significant at 5\% level

Is-Insignificant

Totally experimentally involved six natural commodities ethanol extracts analyzed Minimum Inhibitory Concentration efficiency were presented in table - 2. From the result five different concentrations $(50,100,150,200$ and $250 \mu \mathrm{l}$ ) has been made for this MIC value measurement on each natural commodities such Mayaca, Gooseberry fruit, Garlic extract, Wine, Coconut oil and Pomegranate fruit juice. In account of MIC value was little higher 0.90 followed by pomegranate fruit juice 0.79 and third most efficiency has been noted in wine extract 0.74 in $50 \mu 1$ concentration. In addition this result clearly showed whenever the concentration was maximized; the MIC value was also decreased according to their respective concentrations. Finally, among the six experimental extracts Mayaca and Goosberry fruit extracts showed the similar minimum inhibitory efficiency value been noticed 0.14 (Figure-1\&3). According to the ANOVA analysis incorporated results were represented in table 3 . While, the antifungal activity of Mayaca ehtanolic extract against $C$. albicans confirmed the significant activity at 50 and $100 \mu \mathrm{l}$ concentration $(\mathrm{P}<0.05)$, and it was also been observed in garlic and gooseberry. But in the wine and coconut oil possessed insignificant antifungal activity detected by the ANOVA statistical analysis.

GC-MS analysis of Mayaca revealed that presence of ten compounds (phytochemical constituents) that could contribute the medicinal quality of the plant. The identification $f$ the phytochemical compounds was confirmed based on the peak area and retention time. The active principles with their Retention time (RT), rese. type, Quan ions, Area and Amount/RE peak names are presented in Table and Fig -1 and 2. The first compound identified with a reduction of retention time $(11.746 \mathrm{~min})$ was 1 Propanone -3-chloro-1. While Bis-3, 4-methylenedioxybe Squalene was the last compound which seized at top retention time (28.666min) to identify. The phytochemical identified through GC-MS analysis showed many biological activities relevant to this study are illustrated in figure-2.

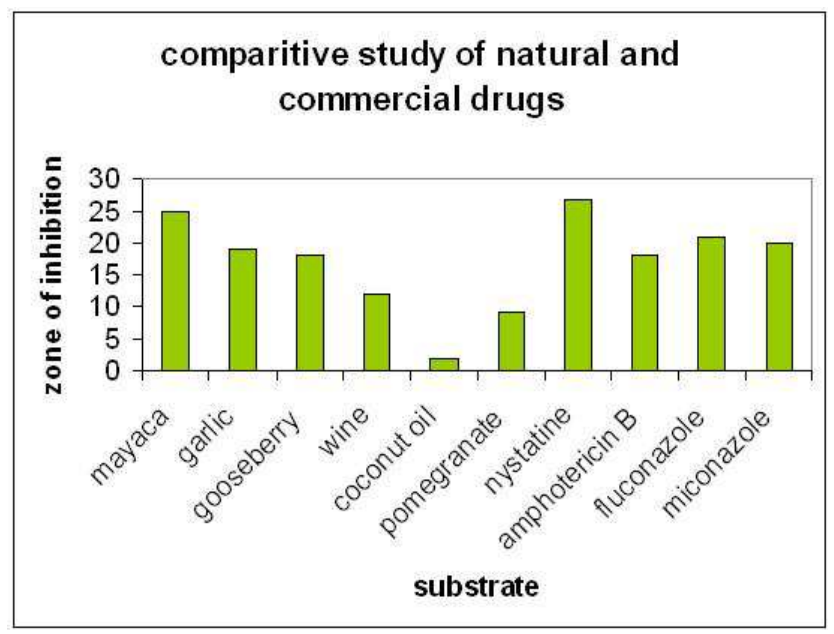

Figure 1. Antifungal activity of natural commodities and commercial drugs against $C$. albicans.

Moreover bioactive phytochemicals were identified peak compound named as 3,4-Dimethyl-2-3-methyl with its Ret time 19.050 followed by second and third peak compounds 
are Diethylpthalate and Bis 3,4 methylene Dioxy accompanied with them responsible RT was 21.004 and
28.666 respectively. Furthermore, sixteen unidentified peak compounds also been observed by GC-MS analysis.

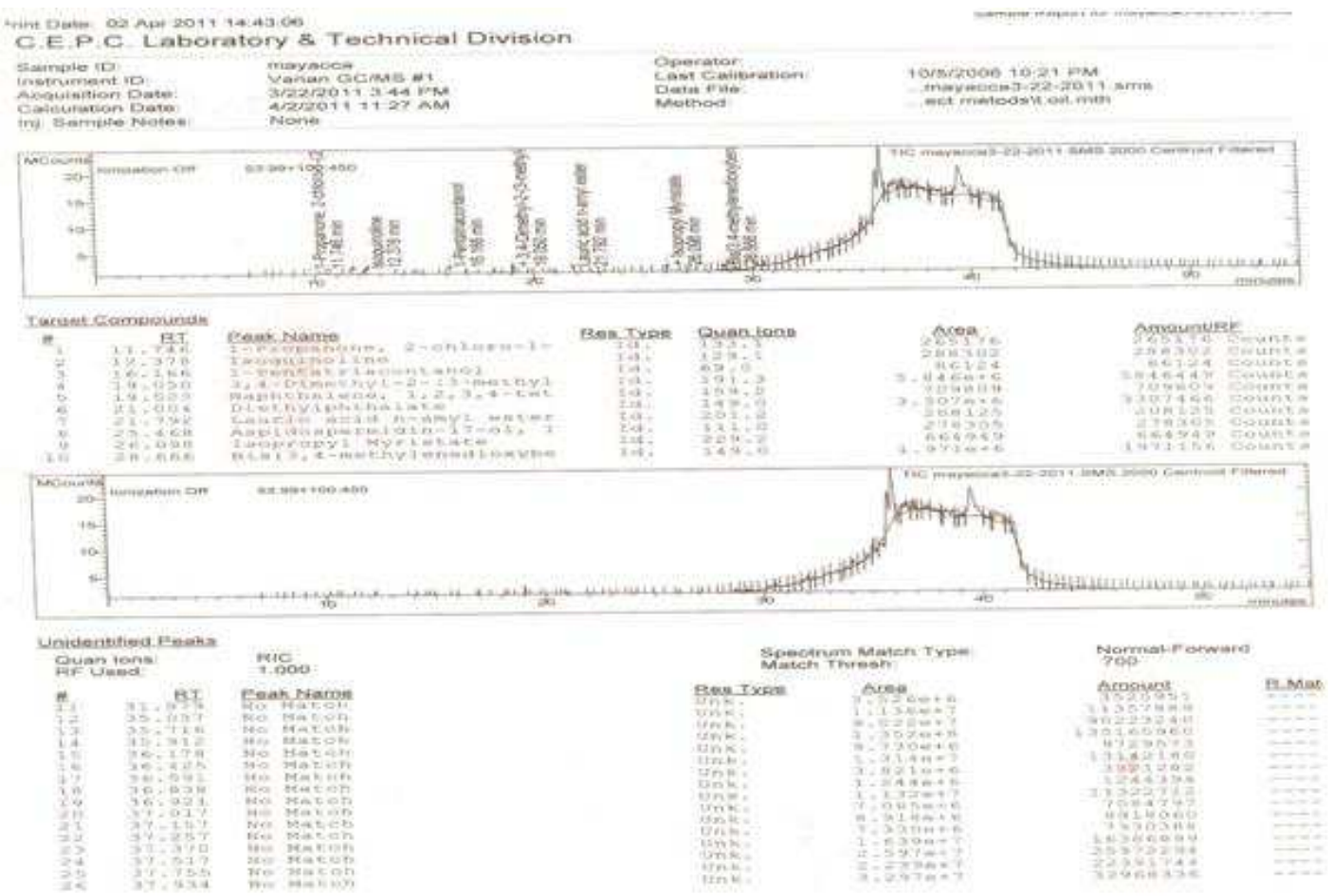

Figure 2. Chromatogram of Mayaca ehtanolic extract by GC-MS analysis.

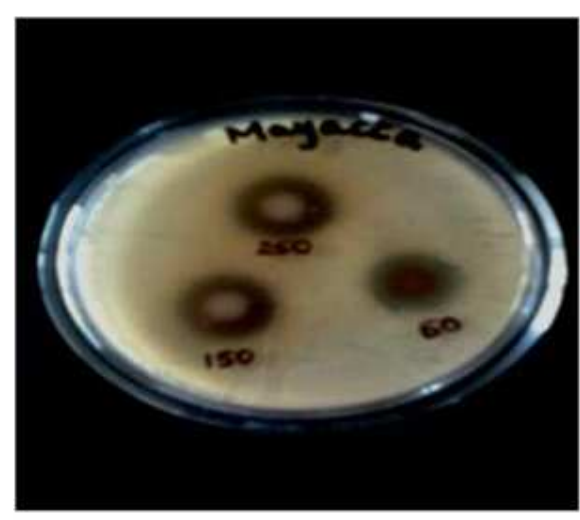

(a)

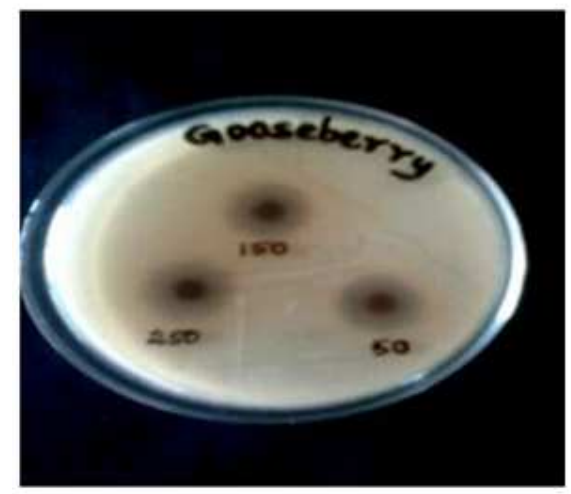

(b)

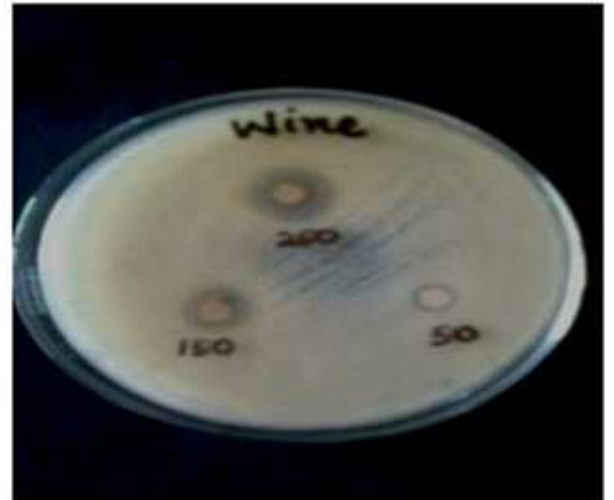

(c)

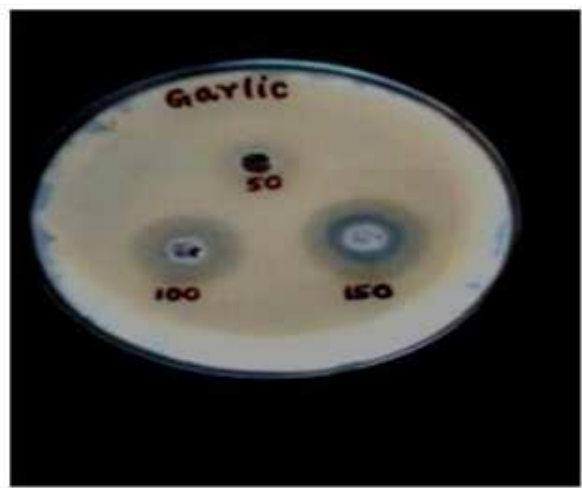

(d)

Figure 3. Showing the clear zone of inhibition against experimental fungus $C$ albicans OF3 A). Mayaca B). Gooseberry C). Wine D). Garlic against. 


\section{Discussion}

In the present study, the result revealed that in natural commodities Mayaca showed high effectiveness against $\mathrm{C}$. albicans. From the statistical report, the result showed that $36 \%$ of babies were infected by oral candidiasis and by using various natural commodities and commercial drugs the candidiosis can be cured. The comparative study stated that among the natural commodities Mayaca showed high effectiveness and Nystatin showed high effectiveness in commercial drugs. The GC MS helped to identify various components present in Mayaca. Ten various phytochemical compounds were identified through GC MS.

From this since showed mayaca showed high effectiveness against Candida albicans, GC MS was performed and identified compounds such as 1-Propanone, 2-chloro-1-(2, 4-dimethylphe; Isoquinoline; 1Pentatriacontanol; 3,4-Dimethyl-2-(3-methyl; Naphthalene, 1, 2, 3, 4-tetrahydro-1,6-dime; Diethylphthalate; Lauric acid n-amyl ester; Aspidospermidin-17-ol, 1-acetyl-19, 21ep; Isopropyl Myristate; Bis[3, 4 methylene dioxy benzoyl] furoxan.

Previously, Duraipandian and Ignacimuthu, ${ }^{15}$ viewed about the fourty medicinal plant extracts against with eleven fungal strains none the plant extract showed remarkable antifungal potent against only the fungi of $\mathrm{C}$. albicans. It was little controversial evidence regarding this research.

Abate screened about 60 different basidiomycetes cultures for antimicrobial secondary metabolites. activity ${ }^{16}$.

Previously, Mwambete, 5 described the agreed view regarding the antimicrobial activity especially the fungal effect against the other different solvent extract such as methanolic and petroleum ether crude extracts of leaves and fruits of $\mathrm{M}$. charantia have adequate antimicrobial activity mainly depends upon the bioactive compounds. Fruits crude extracts possess relatively higher antimicrobial activity compared to leaf crude extract. Nevertheless, mixtures of the fruit and leaf extracts seem to have neither a synergistic nor additive antimicrobial activity on the tested microorganisms ${ }^{17,}{ }^{18}$. This study represents the preliminary report on antimicrobial activity of the crude extracts of $\mathrm{M}$. charantia against both the clinical isolates and reference bacterial strains that are implicated in opportunistic as well as nosocomial infections ${ }^{19}$. Oral candidiasis is defined as an infection of the mucous membrane of the oral cavity caused by yeasts of the genus Candida. The extract of rhizomes of Zingiber officinale has pronounced inhibitory activities against $\mathrm{C}$. albicans. This is comparable with other studies suggesting that different antifungal agents are present in ginger extract ${ }^{20}$

In the past number of researchers studied various kinds of work regarding the antifungal effect of commercialized antibiotics and its susceptibility of Candida albicans to catechin under varying $\mathrm{pH}$ conditions and the synergism of the combination of catechin and antimycotics ${ }^{21}$ (Quiroga et al., 2006). Combined treatment with catechin allows the use of lower doses of antimycotics and induces multiple antifungal effects ${ }^{22}$ (Hirasawa et al., 2004). Banso et al. ${ }^{23,24}$ reported that the antifungal substances contained in the extracts were fungistatic at lower concentrations, while becoming fungicidal at higher concentrations of the extracts. In this result indicate that the MFC of the extracts evaluated were obtained at similar or higher concentrations than in the MIC assays, but not at lower concentration. Omer, ${ }^{25}$ reported recent years, although technology and medicine have developed extensively, some countries have made it obligatory to use natural products for many different purposes due to decrease in natural richness and drawbacks. Like in many other countries, the plants knownby people with health benefits are picked up and used for the treatment of various diseases

\section{Conclusion}

The results concluded from this work showed the Mayaca extracts exhibit antifungal effect against $C$. albicans. In this regard particular comparative efficiency of this ehtanolic extract of mayacca possessed peak activity compared with other five natural commodities as well as four natural drugs.Among the six plants analyzed, Mayaca showed better inhibitory effect against $C$. albicans. GC MS was performed to identify the compounds in Mayaca responsible peak compounds such as 1-Propanone, 2chloro-1-(2, 4-dimethylphe; Isoquinoline; 1Pentatriacontanol; 3, 4-Dimethyl-2-(3-methyl; Naphthalene, 1, 2, 3, 4-tetrahydro-1, 6-dime; Diethylphthalate; Lauric acid n-amyl ester; Aspidospermidin-17-ol,1-acetyl-19,21ep; Isopropyl Myristate; Bis[3, 4 thylenedioxybenzoyl] furoxan.

Further studies are needed to determine the chemical identity of the bioactive imminent compounds responsible for the experimental antifungal activity predominantly antifungal chemical compounds. Natural plant-derived fungicides may be a source of new alternative active compounds, in meticulous with antifungal activity. The elevated fraction of active extracts in the assayed plant extract, selected according to available ethnobotanical data, corroborates the validity of this approach for the selection of plant species in the search for a specific activity.

\section{Acknowledgements}

We grateful to our Malankara Catholic College authorities (Correspondent Fr. Prem Kumar .M.S.W) given encouragement and support for preparation of this research manuscript.

\section{References}

[1] Pei, P.C., Rohani, S. A.H. Yee L.L., Chin L.P., Boon, C.T., Kee, P.N. and Heng, F.S. (2007). Genotyping and drug 
resistance profile of Candida spp. In recurrent and one-off vaginitis, and high association of non $\mathrm{C}$. albicans species with non pregnant status. Infect. Gen. Evol. 7:449-456.

[2] Williams, D.W., Lewis, M.A. (2000). Isolation and identification of Candida from the oral cavity. Oral Dis, 6: 3-11.

[3] Noumi, E., Snoussi, M., Hajlaoui, H., Valentin, E., Bakhrouf, A. (2010). Antifungal properties of Salvadora persica and Juglans regia $1 \mathrm{~L}$. extracts against oral Candida strains. Eur. J. Clin. Microbiol, Infect. Dis. 29:81-88.

[4] Ramawat KG, Sonie KC, Sharma MC (2004). Therapeutic potential of medicinal plants: an introduction. In: Ramawat K.G. (ed) Biotechnology of Medicinal Plants: Vitalizer and Therapeutic. Science Publishers, Enfield, CT, p 1

[5] Mwambete, K D. 2009. The in vitro antimicrobial activity of fruit and leaf crude extracts of Momordica charantia: A Tanzania medicinal plant, Afr Health Sci, 9(1): 34-39.

[6] Muanza, D. N. Kim, B. W Euler K. L. and Williams, L. (1994). Antibacterial and Antifungal Activities of Nine Medicinal Plants from Zaire, Pharmaceutical Biology. 32. 4, 337-345.

[7] Santos, V.R., Pimenta, F.J., Aguiar, M.C., Carmo, do M.A., Naves, M.D. and Mesquita, R.A. (2005). Oral candidiasis treatment with Brazilian ethanol propolis extract. Phytother. Res. 19:652-664.

[8] Silici, S. and Koc, A. N. (2006). Comparative study of in vitro methods to analyse the antifungal activity of propolis against yeasts isolated from patients with superficial mycoses. Lett. Appl. Microbiol. 43:318-324.

[9] Ghasem, Y.B., Ownagh, A.W. and Hasanloei, M. (2007). Antibacterial and antifungal activity of Iranian propolis against Staphylococcus aureus and Candida albicans. Pak. J. Biol. Sci. 10:1343-1345.

[10] Quiroga, E.N., Sampietro, D.A., Soberon, J.R., Sgariglia, M.A.and Vattuone, M.A. (2006). Propolis from the northwest of Argentina as a source of antifungal principles. J. Appl. Microbiol, 101:103-110.

[11] Hornby, J.M., Jensen, E.C., Lisec, A.D., Tasto, J.J. Jahnke, B., Shoemaker, R., Dussault, P., and Nickerson, K.W. (2001). Quorum sensing in the dimorphic fungus Candida albicans is mediated by farnesol. Appl. Environ Microbiol, 67:29822992.

[12] Martin, M.V. (1999). The use of fluconazole and itraconazole in the treatment of C. albicans infections: a review. Journal of Antimicrobial Chemotherapy, 44:429-437.

[13] Lyudmila, B.G.G, Rossen, N., Sirigan, D, Elena, L, Nikolov, K., Ivan, M.,Zacharii, K. 2005. Activity of Bulgarian propolis asainst 94 Helicobacter pylori strains in vitro by agar well diffusion agar dilution and disc diffusion methods. J.Med. Micobiol, 54: 481-483.

[14] Chabra SC, Shao JF, Mshiu EN. Antifungal activity amomg the traditionally used herbs in Tanzania. The Dar Medical Journal. 1982; 9: 68-73.

[15] Duraipandiyan, N. Ignacimuthu, S. 2011. Antifungal activity of Traditional medicinal plants for Tamilnadu, India. Asian Journal of Tropical Medicin, 8204-8215.

[16] Rana P. S. and D. A. Jain. 2011. Screening for Anti-Fungal Activity of Some Medicinal Plant Species from North India, Asian Journal of Biochemical and Pharmaceutical Research, 2, 1: 283-291.

[17] Rana P. S. and D. A. Jain. 2011. Screening for Anti-Fungal Activity of Some Medicinal Plant Species from North India, Asian Journal of Biochemical and Pharmaceutical Research, 2, 1: 283-291.

[18] Srinivasan D, Nathan S, Suresh T, Lakshmana Perumalsamy P. 2001 Antimicrobial activity of certain Indian medicinal plants used in folkloric medicine. J Ethnopharmacol,; 74: 217- 220 .

[19] Jia W, Zhang L. (2005) Challenges and opportunities in the Chinese herbal drug industry. In: Demain AL, Zhang L (eds) Natural Products: Drug Discovery and Therapeutic Medicine. Humana, Totowa, NJ, PP 229.

[20] Okubo, S., Toda, M. and Hara, Y. (1991). Antifungal and fungicidal activities of tea extract and catechin against Trichophyton. Nippon Saikingaku Zasshi, 46: 509-514.

[21] Parekh J, Chanda S. (2008). In vitro antifungal activity of methanol extracts of some Indian medicinal plants against pathogenic yeast and moulds.African . J. Biotechnol, 7 (23): 4349-4353.

[22] Portillo A, Vila R, Freixa B, Adzet T, Canigueral S. (2001). Antifungal activity of Paraguayan plants used in traditional medicine. J. Ethnopharmacol, 76: 93-98.

[23] Banso, A., S.O. Adeyemo, and P. Jeremiah. 1999. Antimicrobial properties of Vernonia amygdalina extract. Journal of Applied Science and Management, 3:9-11.

[24] Woodruff, P.W. and Hasseltine, H.C. (1938). Relationship of oral thrush to vaginal mycosis and evidence of each. Amer. J. Obst. Gynaec. 36: 467.

[25] Omer E., 2006. Antibacterial and antifungal activity of ethanolic extracts from eleven spice plants, Biologia Bratislava, 61/3: 275-278. 